\title{
Amniotic band syndrome associated with extremely severe atypical clefts of the orofacial region
}

\author{
Mekonen Eshete $^{1} \cdot$ Muhidin Abdo Banko $^{2} \cdot$ Abiye Hailu $^{1,3} \cdot$ Abeje Brhanu $^{4} \cdot$ Peter Mossey $^{5} \cdot$ Azeez Butali $^{6}$
}

Received: 24 April 2021 / Accepted: 2 July 2021 / Published online: 18 August 2021

(c) The Author(s) 2021

\begin{abstract}
Amniotic band syndrome (ABS) is not a commonly seen birth defect; however, it may have the potential to be severe and life-threatening requiring adequate attention. We present a severe case of amniotic band syndrome which encircled the head of the neonate tightly causing severe deformity. In this case report, the amniotic band encircled the head causing a severe bilateral Tessier 7 cleft. To our knowledge, this is the most severe type of ABS reported in the literature thus far.

Level of evidence: Level V, risk / therapeutic study.
\end{abstract}

Keywords Amniotic band syndrome · Transverse orofacial clefts $\cdot$ Tessier 7

\section{Introduction}

Amniotic band syndrome (ABS) is an unusual and poorly understood congenital anomaly which might be the cause of multiple fetal anomalies [1]. ABS can trap any part of the fetus during intrauterine life causing various birth defects. These range from minor constriction rings to the extremities to severe anomalies that may be incompatible with life [2]. The incidence of this anomaly is not precisely known, but there are reports suggesting that it occurs between 1 in 1200 and 1 in 15,000 live births [3]. The deformity associated with ABS depends on the area it involves. If it involves the orofacial region, atypical clefts

Mekonen Eshete

Mekonene@yahoo.com

1 Surgical Department, School of Medicine, College of Health Sciences, Addis Ababa University, Addis Ababa, Ethiopia

2 Department of Obstetrics and Gynecology, Ethio Tebib Hospital, Addis Ababa, Ethiopia

3 Plastic and Reconstructive Surgery Department, Hospital Medical College, YekatitAddis Ababa, Ethiopia

4 Department of Surgery, Plastic and Reconstructive Surgery Unit, St. Paul's Millennium Medical College, Addis Ababa, Ethiopia

5 Department of Orthodontics, University of Dundee, Scotland, UK

6 Department of Oral Pathology, Radiology and Medicine, College of Dentistry, University of Iowa, Iowa City, IA, USA of all types may occur, also affecting the eyes, nose, and ears. According to Seeds et al. [3], fetal malformations associated with this anomaly are grouped into three main categories: limb defects, craniofacial defects, and visceral defects. Additionally, a combination of anomalies may exist, similar to what we have seen in our case. The limb abnormalities include constriction rings, amputations, pseudo syndactyly, abnormal dermatoglyphs, and clubbed feet. The craniofacial abnormalities associated with ABS include anencephaly, encephalocele, cleft lip and palate, nasal deformities, asymmetrical microphthalmia, and abnormal calcification of the skull. Omphalocele and gastroschisis are the most common visceral abnormalities. In our practice, we have seen patients with different malformations associated with ABS; here, we demonstrate one specific case with unusual presentations. We believe this case report will contribute to the existing knowledge in the etiopathology of the anomaly. This case report is part of a bigger study entitled The Role of Genetics and Environmental Factors in the Etiology of Orofacial Clefts in the Ethiopian Population for which ethical approval was obtained from the Institutional Review Board at the Addis Ababa University College of Health Sciences (IRB approval number: 003/10/surg) and renewed yearly. In addition, written informed consent was obtained from the parents. 


\section{Case report}

This case report is of a female neonate born at term; the second child in four pregnancies (one previous still birth, a missed abortion, and one healthy child) in a healthy 28 -yearold mother. There was no history of trauma or drug intake during pregnancy. History of malformations in the family and consanguinity were both absent. The mother received regular antenatal care and had favorable course until term labor. As part of the antenatal care, ultrasound examination was performed at different stages of the pregnancy, which did not reveal any fetal malformations. Delivery occurred at 41 weeks and 2 days gestational age by cesarean section following the indication of prolonged active first stage of labor and a previous cesarean section scar. The outcome of the pregnancy was a female neonate with a birth weight of $3120 \mathrm{~g}$ and Apgar scores of 8 in the first minute and 9 after $5 \mathrm{~min}$. During delivery, the fetal chorionic membrane encircled the head above the ear going through the mouth as seen in Fig. 1. It was detached from the neonate after clamping and ligation.

Initial examination showed multiple malformations consisting of a severe bilateral transverse cleft of the orofacial region extending posteriorly and joined with the other side

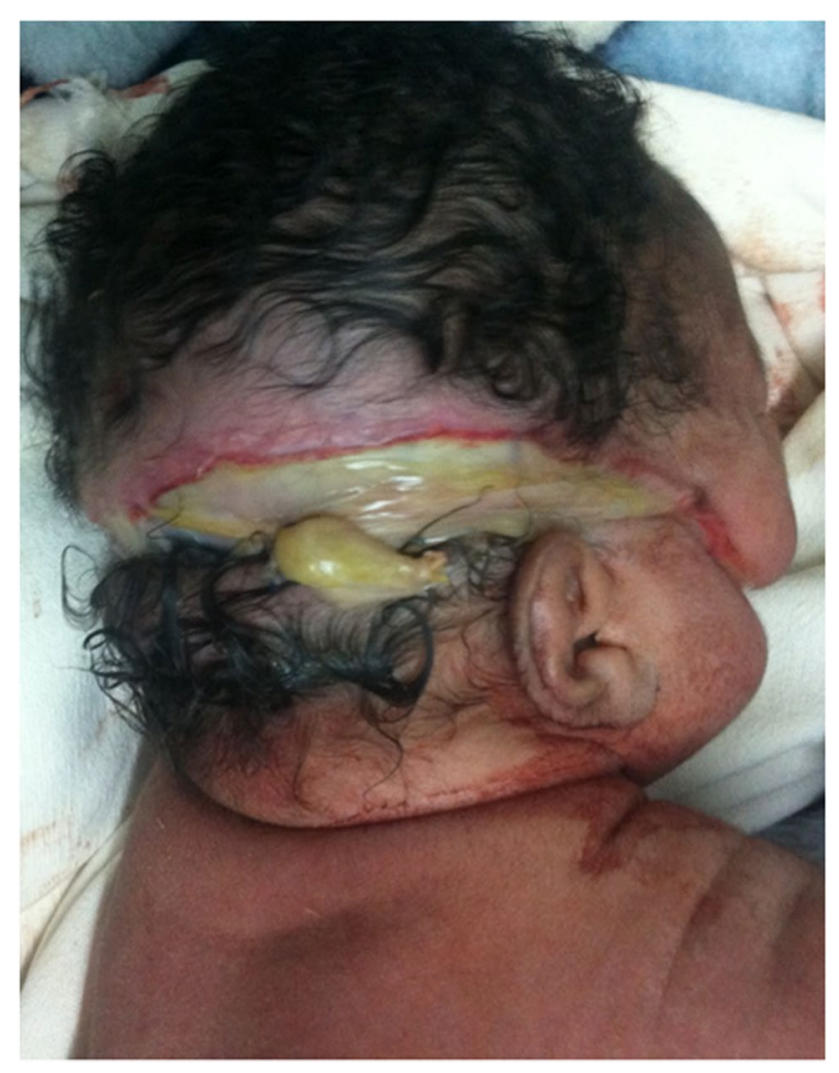

Fig. 1 Amniotic band encircling the head with a deep furrow on the scalp (Figs. 2 and 3) and two deep indentations on the lower lip simulating bilateral incomplete lower lip cleft (Fig. 3). There were no other clinically identifiable anomalies. Karyotype was normal female (46, XX).

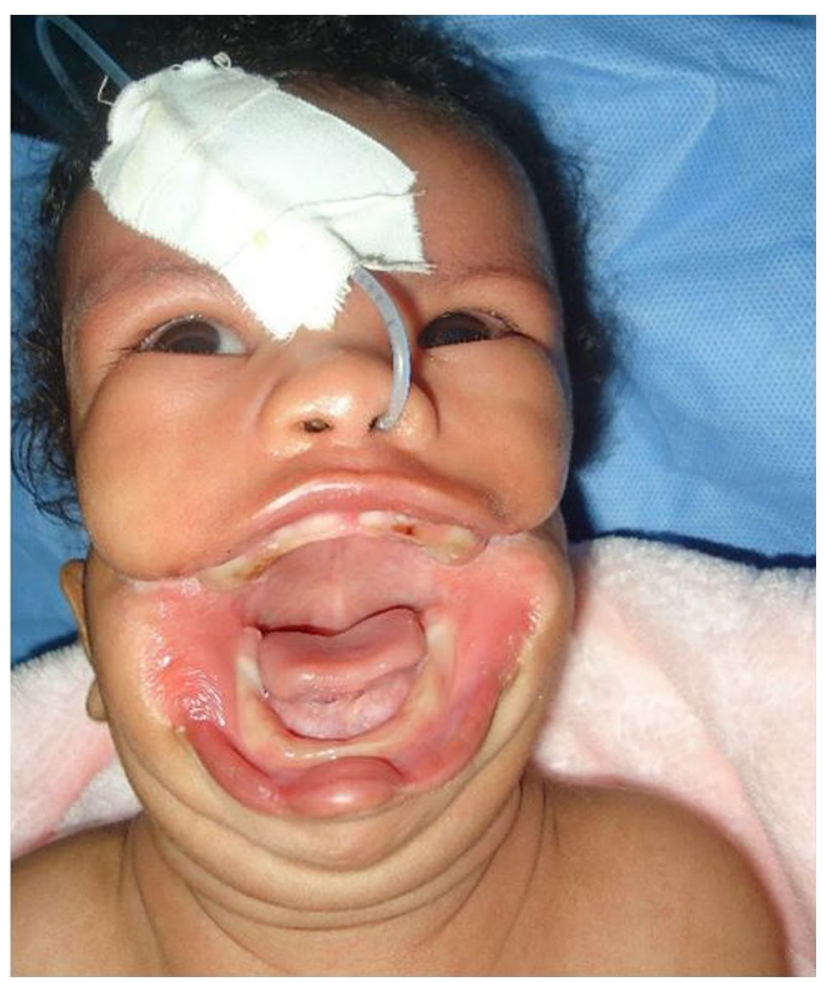

Fig. 2 Front view of the deformity

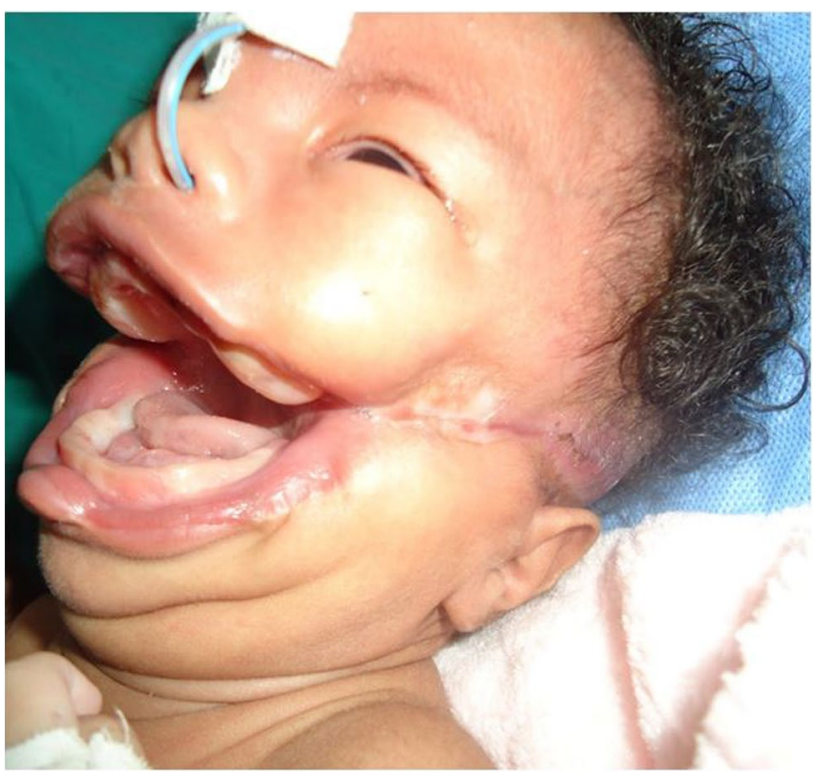

Fig. 3 Side view of the deformity 
The patient was referred to Yekatit 12 Hospital Medical College Plastic and Reconstructive Surgery Department following a telephone communication. At presentation, physical examination revealed a 1-week-old neonate who was alert, with nasogastric tube in situ. There was a severe bilateral transverse cleft simulating Tessier 7 cleft and a circumferential scar on the scalp with a palpable defect of the temporal and occipital bones. There were deep indentations on the lower lip causing bilateral incomplete cleft. The patient's mother had reported her eyes as only partially closed during sleep. Both ultrasound and MRI of the central nervous system and abdominal organs showed normal anatomy of examined organs, with the exception of a defect of the temporal and occipital bones. Basic hematologic tests were also within normal ranges. We sought the support of a pediatric anesthesiologist and operated the child with the main aim of improving feeding, speech, and appearance.

Plastic and reconstructive surgeons operated on the neonate using Butow and Botha's four layered techniques with very good outcome [4] at the age of 3 weeks (Fig. 4). This was the youngest patient with orofacial clefts we have operated on with the main intent to improve feeding. Two additional follow-up surgeries were performed at the age of 2 and 4 years old to remove the indentations on the lower lip and improve the side scars. The child is currently 6 years old, has intelligible speech and good appearance, is normal, and is appropriate for age mental and psychomotor development. She cannot close her eyes forcefully or completely during sleep, but there is no corneal exposure. She has no saliva drooling, can eat without difficulty, and only has mild drooling with drinking (Fig. 5). There is a palpable bony defect on the temporal and occipital regions, but it is getting narrower.

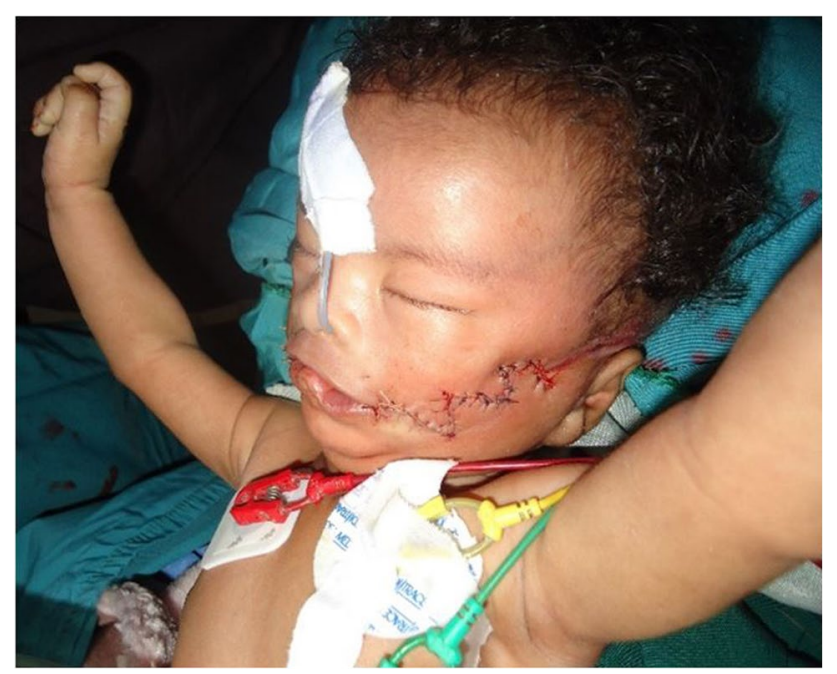

Fig. 4 Immediate postoperative photo

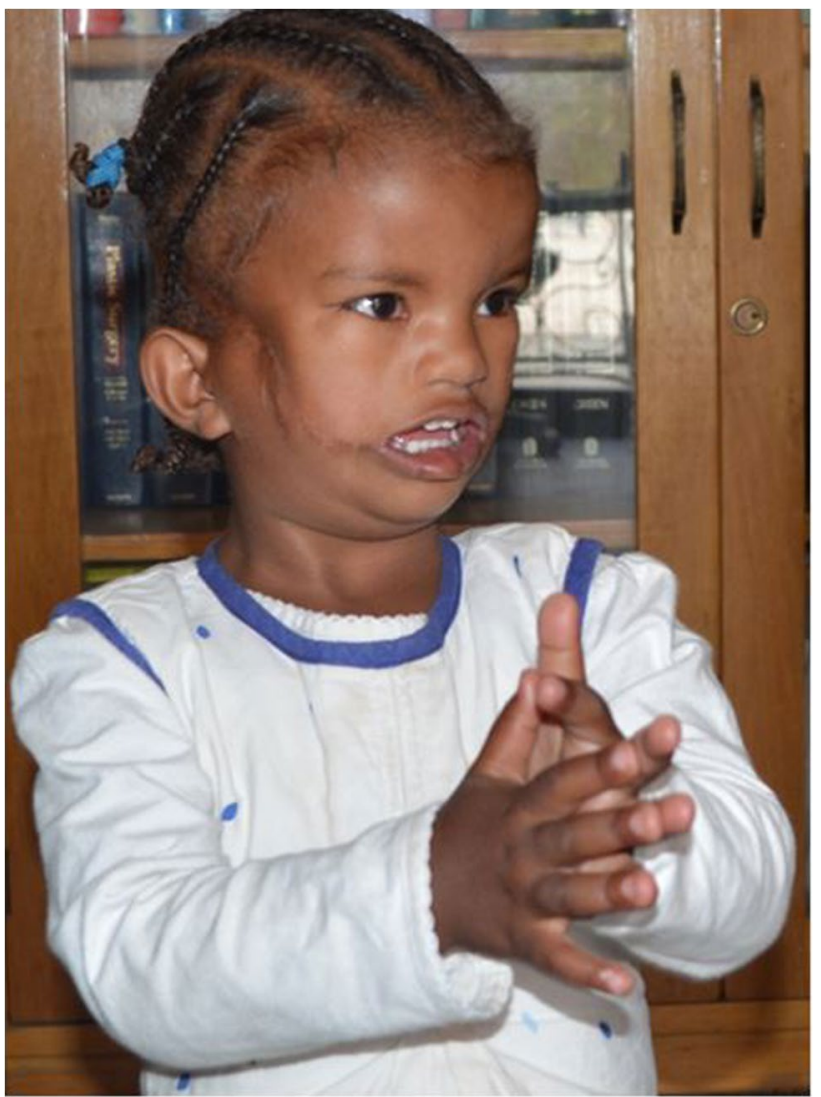

Fig. 5 Follow-up photo

\section{Discussion}

We present a severe case of ABS-related facial cleft, an unusual case of a bilateral transverse facial cleft (bilateral Tessier 7). At delivery, a tight amniotic band was found encircling the head passing through the mouth and above the ears. There were indentations on the lower lip causing an incomplete bilateral lower lip cleft. To our knowledge, this is the severest type of Tessier No. 7 cleft reported so far.

The etiology and pathogenesis of ABS is unknown. It is a sporadic disorder [5] which is characterized by constricting rings, acrosyndactyly, and often amputations of the extremities of neonates. Hippocrates recognized this condition as early as $300 \mathrm{BC}$ [6]. He suggested that extrinsic pressure from a ruptured amniotic membrane result in band formation or digital amputation.

After examining many placentae and infants born with amniotic band syndrome, Richard Torpin [7] reintroduced the extrinsic theory (Hippocrates' theory). He noticed lack of a complete amniotic lining in the placentae of neonates born with ABS. Torpin also observed strands of amnion around constricting rings of the digits, and binding strands at the tips of limbs with acrosyndactyly. Similarly, we observed amniotic bands encircling the head of the neonate 
and divided after ligation as shown in Fig. 1. This presents additional strong evidence supporting Hippocrates' suggestion regarding the role of ruptured amniotic band in the occurrence of this disorder. Unfortunately, the placenta of our patient was not examined properly to support Torpin's findings. According to Torpin, intrauterine trauma could be the cause of premature rupture of the membranes. There was no history of intrauterine trauma in our case.

Matic and Komazec [8] stated that most cases of ABS are not of genetic origin with no recurrence in siblings or children of affected adults. There was no recurrence in this case study; the patient has an older sister and a younger brother without ABS in either of them.

Although there is no firm evidence of definitive causality, there are studies reporting a connection between ABS and the mother's age especially primiparas under the age of $25[9,10]$, birth prematurity [11], abdominal trauma [10], unsuccessful abortion [12], intrauterine contraception [13], cerclage [14], and chorionic villus sampling [12]. Having little evidence of definite causality for any of the above listed factors, many investigators consider the occurrence of ABS as sporadic with no strong risk factors and gender prevalence [15]. The parents of our patient did not present any of these listed risk factors.

Another attempt to explain the cause of this anomaly was done by Streeter in 1930 [16]. He proposed the intrinsic model suggesting that both the anomaly and the fibrous bands have a common origin as caused by a deviation in the normal development of the early embryo's germinal disc.

The morphology of the deformity on the developing fetus could be determined by the severity of the band compression. As shown in Fig. 2, the deep and circumferential amniotic band caused severe and atypical transverse facial cleft involving deeper structures: temporal and occipital bones and also the facial nerve. The child currently cannot close her eyes tightly. On the other hand, when the bands are superficial, only skin indentations occur.

Prenatal ultrasound examination can sometimes detect amniotic bands, but more often malformations associated with ABS, oligoamnios, and reduction of fetal movements [17]. According to Merrimen [18], it is possible to detect ABS as early as 12 weeks of gestational age. A second trimester routine ultrasound examination can detect most of the defects associated with ABS [17]. In our case, with regular antenatal care inclusive of ultrasound examination, neither amniotic band abnormality nor malformation was detected. This is an area needing improvement in the care of pregnant women in a resource-limited setting. Procuring the latest three-dimensional and four-dimensional ultrasound machines and enabling the professionals to use them is equally important.

The management of children born with birth defects in low- and middle-income countries such as Ethiopia is not simple. It should start with parental counselling inclusive of psychological support to the parents, feeding advice, emphasizing the need for multiple surgeries, and thorough follow-up. The surgical procedure for patients with ABS may range from minor to complex and the outcomes depend on the severity of the deformity. In our case, the surgical management was challenging because of the need to operate at an early neonatal age. Our patient's surgical management is not yet complete, as she will need some additional follow-up surgeries to improve the indentations on the temple regions. The bony defect on the temporal and occipital regions is narrowing but might necessitate bony reconstruction in the future should the bony defect remain open. There are some signs of facial nerve involvement such as the inability to close eyes forcefully, nor during sleep, and mild drooling while drinking.

\section{Conclusions}

Amniotic band syndrome is a rare birth defect and has the capacity to threaten life or cause a severe incapacitating deformity. This anomaly may be detected as early as the first trimester of pregnancy if the appropriate facility and expertise is available. Measures can also be taken when the deformity is not compatible with life. Therefore, we need to improve the antenatal follow-up at our institutions; this might include procuring the latest advanced ultrasound machines and providing education for our medical professionals. Furthermore, additional education is needed in the community for the improvement of attitude, beliefs, and understanding of birth defects so that those affected will receive timely treatment and reduce stigma.

Acknowledgements Transforming Faces, Smile Train, and Yekatit 12 Hospital Medical College are acknowledged for supporting the treatment of this child and other children and adults born with orofacial clefts. The authors would like to thank Dr Larry Hollier and Abel Smerica for reading our case report and providing valuable comments.

Funding This study was supported by Grant R00-DE022378 (Azeez Butali) and subaward to Mekonen Eshete and by Smile Train.

\section{Declarations}

Ethics approval All procedures performed in studies involving human participants were in accordance with the ethical standards of the institutional and/or national research committee and with the 1964 Helsinki Declaration and its later amendments or comparable ethical standards. Ethical approval was obtained from the Institutional Review Board at the Addis Ababa University College of Health Sciences (IRB approval number: 003/10/surg) and renewed yearly.

Consent to participate Written informed consent was obtained from the parents. 
Consent for publication Written informed consent was obtained from the parents including the use of the child's photos.

Conflict of interest Mekonen Eshete is a member of the Smile Train Research and Innovation Advisory Council. Muhidin Abdo Banko, Abiye Hailu, Abeje Brhanu, Peter Mossey, and Azeez Butali declare no competing interests.

Open Access This article is licensed under a Creative Commons Attribution 4.0 International License, which permits use, sharing, adaptation, distribution and reproduction in any medium or format, as long as you give appropriate credit to the original author(s) and the source, provide a link to the Creative Commons licence, and indicate if changes were made. The images or other third party material in this article are included in the article's Creative Commons licence, unless indicated otherwise in a credit line to the material. If material is not included in the article's Creative Commons licence and your intended use is not permitted by statutory regulation or exceeds the permitted use, you will need to obtain permission directly from the copyright holder. To view a copy of this licence, visit http://creativecommons.org/licenses/by/4.0/.

\section{References}

1. Editoreal A (1973) bands. Br Med J (Clin Res Ed) 3:651-652

2. AleksandraM J (2009) Amnioticbandsyndrome Acta MedMed 48:44-48

3. Seeds EA (1983) Br Med J (Clin Res Ed) 286:919-920

4. Khorasani* H, Boljanovic S, Jacobsen LP, Mary Amma Kjærulff Knudsen (2019) Surgical management of the Tessier 7 cleft: a review and presentation of 5 cases. JPRAS Open. 22: 9-18

5. Quintero RA, MW, Phillips J, Kalter CS, Angel JL (1997) In utero lysis of amniotic bands. Ultrasound Obstet Gynecol. 10(5): 316-320

6. Twee DO (2006) Streeter dysplasia. e-medicine, December
7. Torpin R (1965) Amniochorionic mesoblastic fibrous strings and amnionic bands associated constricting fetal malformations or fetal death. Am J Obst Gynec 91(1):65-75

8. Komaze AMAJ (2009) Amniotic band syndrome. Acta Medica Medianae. 48

9. Werler MM, LC, Mitchell AA (2003) Epidemiologic analysis of maternal factors and amniotic band defects. Birth Defects Res A Clin Mol Teratol 67(1): 68-72

10. Dyer JA, CS (2005) Acquired raised bands of infancy: association with amniotic bands. Pediatric Dermatology 22(4): 346-349

11. Clarke JC, BE, Salonen DC (2000) Amniotic band syndrome. Can Assoc J Radiol 51(2): p. 134-136

12. Ferns S, SR (2001) Narayanan P, Bhat BV, Limb amputation defects in utero-a case report. J Anat Soc India 50(2): 156-158

13. Burk CJ, AC, Connelly EA (2007) Ehlers-Danlos syndrome type IV: keloidal plaques of the lower extremities, amniotic band limb deformity, and a new mutation. J Am Acad Dermatol 56: 553-554

14. Kanit H, AD, Dicle N, Ugurel G (2001) Amniotic band syndrome after cervical cerclage. Perinatoloji Dergisi. 9: 1-3

15. Poeuf B, SP, Magalon G (2008) Amniotic band syndrome. Chir Main 27: s136-47

16. Streeter GL (1930) Focal deficiencies in fetal tissues and their relation to intrauterine amputations. Contrib Embryol Carnegie Inst 22: $1-4$

17. Allen LM (2007) Constriction rings and congenital amputations of the fingers and toes in a mild case of amniotic band syndrome. J Diagnost Med Sonogr 23: 280-285

18. Merrimen JL, MP, Bendor-Samuel RL, Schmidt MH, Fraser RB (2006) Congenital placental-cerebral adhesion: an unusual case of amniotic band sequence. Case report. J Neurosurg. 104: 352-355

Publisher's note Springer Nature remains neutral with regard to jurisdictional claims in published maps and institutional affiliations. 